\title{
Effects of the Chemical Chaperone 4-Phenylbutylate on the Function of the Serotonin Transporter (SERT) Expressed in COS-7 Cells
}

\author{
Masayuki Fujiwara ${ }^{1}$, Hikaru Yamamoto ${ }^{1}$, Tatsuhiro Miyagi ${ }^{1}$, Takahiro Seki ${ }^{1}$, Shigeru Tanaka ${ }^{1}$, Izumi Hide ${ }^{1}$, \\ and Norio Sakai ${ }^{1, *}$ \\ ${ }^{1}$ Department of Molecular and Pharmacological Neuroscience, Institute of Biomedical \& Health Sciences, \\ Hiroshima University, 1-2-3 Kasumi, Minami-ku, Hiroshima 734-8551, Japan
}

Received August 29, 2012; Accepted March 27, 2013

\begin{abstract}
The serotonin transporter (SERT) is involved in various psychiatric disorders, including depression and autism. Recently, chemical chaperones have been focused as potential therapeutic drugs that can improve endoplasmic reticulum (ER) stress-related pathology. In this study, we used SERT-transfected COS-7 cells to investigate whether 4-phenylbutylate (4-PBA), a chemical chaperone, affects the membrane trafficking and uptake activity of SERT. Treatment with 4-PBA for $24 \mathrm{~h}$ dose-dependently increased the uptake activity of SERT. In accordance with increased SERT activity, the expression of maturely glycosylated SERT was increased, while the expression of immaturely glycosylated SERT was decreased. This finding suggests that 4-PBA increased the functional SERT with mature glycosylation via accelerating its folding and trafficking. 4-PBA also increased the activity of the C-terminus-deleted mutant SERT (SERT $\triangle \mathrm{CT}$ ), which was stacked in the ER, and decreased SERT $\triangle \mathrm{CT}$-induced ER stress, further supporting the idea that 4-PBA acts as a chemical chaperone for SERT. Imaging studies showed that fluorescencelabeled SERT was gradually and significantly translocated to the plasma membrane by 4-PBA. These results suggest that 4-PBA and related drugs can potentially affect serotonergic neural transmission by functioning as chaperones, thereby providing a novel therapeutic approach for SERT-related diseases.
\end{abstract}

[Supplementary materials: available only at http://dx.doi.org/10.1254/jphs.12194FP]

Keywords: serotonin transporter, chemical chaperone, membrane trafficking, ER stress

\section{Introduction}

Serotonin (5-HT) is a monoamine neurotransmitter that participates in various neural functions, including emotion, sleep, feeding, movement, and temperature control (1). In contrast to the diversity of 5-HT receptors, the serotonin transporter (SERT) is considered to be the only membrane protein that plays an important role in terminating serotonergic neural transmission through the re-uptake of 5-HT into presynaptic terminals $(2,3)$. It is well known that SERT is the target of antidepressants, including selective 5-HT-reuptake inhibitors (SSRI) and tricyclic antidepressants $(4-6)$. Therefore, SERT has

*Corresponding author. nsakai@hiroshima-u.ac.jp

Published online in J-STAGE on May 16, 2013

doi: 10.1254/jphs.12194FP been thought to be a key molecule that is involved in the pathogenesis of mood disorders. Polymorphism studies of the SERT gene revealed that SERT is associated with anxiety and vulnerability to stress $(7,8)$. In addition, missense mutations in the coding region of the SERT gene were identified in families with autism and obsessive-compulsive disorder $(9-11)$. These findings emphasize that SERT is a molecule that is widely related to various neuropsychiatric disorders, including autism, mood disorders, anxiety disorders, and other stress-related diseases.

After receptors and transporters, including SERT, are translated in the endoplasmic reticulum (ER), they are commonly folded into a trans-membrane conformation with the assistance of molecular chaperones. These membrane proteins also undergo glycosylation via the Golgi apparatus during their trafficking from the ER to 
the plasma membrane. In the case of SERT, putative glycosylation sites exist in the second extracellular loop (12). First, SERT becomes immaturely glycosylated by the addition of a mannose in the ER, and then it becomes maturely glycosylated in the Golgi apparatus before it is transferred to the plasma membrane $(13-15)$. Accumulating evidence reveals that the SERT C-terminus plays essential roles in its glycosylation and membrane trafficking because C-terminus-deleted SERT is not expressed in the plasma membrane and does not function as a proper transporter $(16-18)$.

When the proper process for glycosylation and protein folding is impaired, membrane proteins accumulate in the ER, which results in cellular stress called ER stress. It is well known that excess ER stress causes apoptosis. In the central nervous system, ER stress occurs in ischemia, aging, and neurodegeneration, which are all conditions that trigger the accumulation of misfolded proteins $(19-21)$.

Chemical chaperones are drugs that can assist the folding of membrane proteins and accelerate their membrane trafficking. Therefore, chemical chaperones are a focus as potential therapeutic drugs that can improve ER stress-related pathology, including cystic fibrosis and diabetes mellitus $(22-24)$. To date, 4-phenylbutylate (4-PBA), tauroursodeoxycholic acid (TUDCA), trimethylamine $\mathrm{N}$-oxide (TMAO), and trehalose are known to function as chemical chaperones $(25-29)$. Among them, 4-PBA has been reported to improve the membrane trafficking of mutant CFTR (cystic fibrosis transmembrane regulator), which is found in certain families with cystic fibrosis (27). Additionally, 4-PBA alleviates ER stress induced by the Pael receptor, which is thought to be accumulated in PARK2, one type of inherited Parkinson's disease (26). Furthermore, 4-PBA can reduce the area of damage due to ischemia in a rat model of cerebral infarction (30).

Previously, we examined whether proteasome inhibition affects the uptake activity and membrane trafficking of SERT (18). We found that proteasome inhibition caused SERT to be retained in the ER, which resulted in ER stress due to impaired membrane trafficking. In addition, in this state, the expression of maturely glycosylated SERT in the plasma membrane is reduced, thereby causing an attenuation of SERT uptake activity. These results suggest that a drug, which can accelerate the membrane trafficking of SERT and increase its expression in the plasma membrane by assisting its folding, could alter serotonergic neural transmission by increasing the 5-HT content at pre-synaptic terminals and subsequent 5-HT release.

Based on these background data, we focused on chemical chaperones, and in this study, we especially focused on 4-PBA. We examined whether this drug can affect the regulation and function of SERT using SERT-transfected COS-7 cells.

\section{Materials and Methods}

\section{Materials}

Glass-bottom culture dishes were purchased from MatTek Corporation (Ashland, OR, USA). Dulbecco's modified Eagle's medium (DMEM), fetal bovine serum (FBS), and penicillin/streptomycin mixture were purchased from Life Technologies Japan (Tokyo), Biowest (Rue de la Caille, France), and Nacalai Tesque (Kyoto), respectively. An anti-DYKDDDDK tag mouse monoclonal antibody was purchased from Wako Pure Chemical Industries, Ltd. (Osaka). The anti-KDEL mouse IgG antibody and HRP-conjugated anti-mouse IgG antibody were purchased from Jackson ImmunoReseach (West Grove, PA, USA). 4-Phenylbutylate (4-PBA) and butylate were obtained from LKT Laboratories (St. Paul, MN, USA) and Wako Pure Chemical Industries, Ltd. (Osaka), respectively. $\left[{ }^{3} \mathrm{H}\right] 5-\mathrm{HT}(370 \mathrm{GBq} / \mathrm{mmol})$ was purchased from PerkinElmer (Waltham, MA, USA). All other chemicals were of analytical grade.

\section{Generation of plasmids and adenoviral vectors}

A plasmid that can express FLAG-tagged rat SERT in mammalian cells was constructed as described previously (31). Briefly, a rat SERT cDNA fragment with MunI sites on both the $5^{\prime}$ and the $3^{\prime}$ ends was obtained by PCR using the full-length rat SERT cDNA as a template. PCR products were subcloned into the pTB701FL expression plasmid, which can add FLAG amino acid residues to the N-terminus of the target protein. The plasmid is designated here as pFLAG-SERT. A plasmid that can express a mutated form of SERT that lacks the C-terminus was made as described previously (18). This plasmid is designated here as pFLAG-SERT $\triangle \mathrm{CT}$.

We constructed two types of adenoviral vectors to express FLAG-SERT under the regulation of tetracycline $(32,33)$. The first vector expresses the tetracycline transactivator (tTA) under the control of the cytomegalovirus (CMV) promoter (Ad-CMV-tTA). The second vector expresses FLAG-SERT under the control of the TetOp minimal promoter, which is activated by tTA. Use of the first and second vectors in combination enables cell type-specific expression of FLAG-SERT. In the presence of tetracycline, the expression of FLAGSERT is arrested (tet-off system).

To generate an adenoviral vector that can express FLAG-SERT under the control of the TetOp promoter (Ad-TetOP-FLAG-SERT), the TetOp promoter was digested with XhoI and HindIII from the pTet-Splice 
plasmid (Invitrogen, Carlsbad, CA, USA) and subcloned into the XhoI/HindIII site in the pShuttle plasmid (Stratagene, La Jolla, CA, USA). The new plasmid was designated TetOp-pShuttle. FLAG-SERT cDNAs containing a polyadenylation signal were amplified by the polymerase chain reaction (PCR) using the pFLAGSERT plasmid as a template. The PCR products were subcloned into the downstream region of the TetOp promoter in the TetOp-pShuttle vector, which was digested with HindIII and blunted by a DNA blunting kit (Takara Bio, Otsu). The new plasmid was designated pTetOp-FLAG-SERT.

Ad-CMV-tTA and Ad-TetOp-FLAG-SERT were constructed using the AdEasy adenoviral vector system (Stratagene) according to the manufacturer's protocol. Briefly, the shuttle vector was recombined with pAdEasy-1, which is an adenoviral backbone cosmid vector, in the E. coli strain BJ5183. The recombinant adenoviral genome was digested from the cosmid vector by $P a c \mathrm{I}$ and transfected into HEK293 cells, which stably express the E1 gene and produce the E1 gene-deleted adenoviral vector. Proliferated adenoviral vectors were extracted from HEK293 cells and concentrated by cesium chloride ultracentrifugation.

A plasmid that can express HaloTag (HT)-fused FLAG-SERT under the control of the tet-off system was made as follows: The HT expression vector, pHaloTag7, was purchased from Promega (Madison, WI, USA). A cDNA fragment encoding FLAG-SERT, obtained by PCR using the full-length rat SERT cDNA as a template, was subcloned into the AroH51 site of pHaloTag7. The resulting plasmid was designated pFLAG-SERTHaloTag7. A cDNA fragment encoding the FLAGSERT-HT, obtained by PCR using pFLAG-SERTHaloTag7 as a template, was subcloned into the blunted HindIII site of the TetOp-pShuttle. The resulting plasmid was designated pTetOp-FLAG-SERT-HT.

\section{Cell culture and transfection}

COS-7 cells and HEK293 cells were cultured in DMEM supplemented with heat-inactivated FBS (10\%), penicillin $(100 \mathrm{unit} / \mathrm{ml})$, and streptomycin $(100 \mu \mathrm{g} / \mathrm{ml})$. Culturing was performed in a humidified atmosphere containing $5 \% \mathrm{CO}_{2}$ at $37^{\circ} \mathrm{C}$. For electroporation, pFLAGSERT was transfected into COS-7 cells or HEK293 cells using a Gene Pulser (Bio-Rad, Tokyo) or NEPA21 (NEPA GENE, Chiba) according to the protocol recommended by the supplier. For adenoviral transfection, the cells were infected with two types of adenoviral vectors, Ad-CMV-tTA (MOI of 5) and Ad-TetOp-FLAG-SERT (MOI of 10). Ad-CMV-tTA expresses the tetracycline transactivator (tTA) under the control of the CMV promoter. Expressed tTA activates the TetOp minimal promoter in Ad-TetOp- $\gamma$ PKC-GFP, thereby driving the expression of FLAG-SERT. Additionally, treatment with tetracycline can arrest the expression of the target protein. The experimental procedures are summarized in our previous report $(32,33)$.

\section{$\left.{ }^{3} H\right] 5-H T$-uptake assay}

Approximately $6 \times 10^{6}$ transfected COS-7 cells were equally divided into $24-w e l l$ plates. $\left[{ }^{3} \mathrm{H}\right] 5-\mathrm{HT}$ uptake assays were carried out $48 \mathrm{~h}$ after transfection as described previously $(31,34)$. In brief, the culture medium was removed and replaced with Krebs-RingerHEPES (KRH) buffer. KRH buffer contained the following: $120 \mathrm{mM} \mathrm{NaCl}, 4.7 \mathrm{mM} \mathrm{KCl}, 2.2 \mathrm{mM} \mathrm{CaCl}_{2}$, $25 \mathrm{mM}$ HEPES, $1.2 \mathrm{mM} \mathrm{MgSO}_{4}, 1.2 \mathrm{mM} \mathrm{KH}_{2} \mathrm{PO}_{4}$, and $10 \mathrm{mM}$ glucose, $\mathrm{pH}$ 7.4. After a 15-min pre-incubation period at $37^{\circ} \mathrm{C}$, the cells were incubated for an additional $15 \mathrm{~min}$ in the presence of $\left[{ }^{3} \mathrm{H}\right] 5-\mathrm{HT}$. To prevent the degradation of 5-HT, $100 \mu \mathrm{M}$ pargyline and $100 \mu \mathrm{M} \mathrm{L}-$ ascorbic acid were added to the KRH buffer. The uptake of $\left[{ }^{3} \mathrm{H}\right] 5-\mathrm{HT}$ was stopped by washing three times with cold KRH buffer containing $10 \mu \mathrm{M}$ fluvoxamine, and the cells were then lysed with RIPA buffer (10 mM Tris$\mathrm{HCl}, 1 \% \mathrm{NP}-40,0.1 \%$ SDS, $0.1 \%$ sodium deoxycholate, $150 \mathrm{mM} \mathrm{NaCl}$ and $1 \mathrm{mM}$ EDTA $\mathrm{pH}$ 7.4). The cell extracts were diluted in a scintillation cocktail (Clear-Sol II; Nakalai Tesque, Kyoto), and their radioactivity was measured with a liquid scintillation counter (LSC-5100; Aloka, Mitaka). The results were considered to represent the total 5-HT cellular uptake. $\left[{ }^{3} \mathrm{H}\right] 5$-HT uptake in the presence of $10 \mu \mathrm{M}$ fluvoxamine was also measured and was considered to represent the non-specific 5-HT cellular uptake. The specific 5-HT uptake was obtained by subtracting the non-specific 5-HT uptake from the total uptake amount. The 5-HT uptake assay was conducted at a concentration of $100 \mathrm{nM} 5$-HT. To calculate the uptake per mg of protein in the cells, the concentration of protein in the cell extract was measured using a BCA protein assay kit according to the manufacturer's directions (Pierce Biotech, Rockford, IL, USA). The SERT uptake activity was defined as the amount of $\left[{ }^{3} \mathrm{H}\right] 5-\mathrm{HT}$ uptake per mg of protein.

\section{Western blotting}

Western blotting was performed as previously described (35). Briefly, cells $\left(6 \times 10^{6}\right)$ were harvested and resuspended in homogenate buffer $(250 \mathrm{mM}$ sucrose, $2 \mathrm{mM}$ EDTA, $10 \mathrm{mM}$ EGTA, $20 \mathrm{mM}$ Tris-HCl, $\mathrm{pH}$ 7.4). The cells were then centrifuged at $3000 \mathrm{rpm}$ for $5 \mathrm{~min}$ at $4{ }^{\circ} \mathrm{C}$, and the pellet was resuspended and sonicated in $100 \mu \mathrm{l}$ of homogenate buffer, which contained $1 \%$ Triton-X and protease inhibitors $(200 \mu \mathrm{g} / \mathrm{ml}$ of leupeptin and $1 \mathrm{mM}$ PMSF). The concentration of protein in cell lysates was 
measured using a BCA protein assay kit. The 20 to $50 \mu \mathrm{g}$ of protein in the cell lysate sample was electrophoretically separated by SDS-PAGE and then transferred onto polyvinylidene difluoride (PVDF) filters (Millipore, Billerica, MA, USA). After non-specific binding sites on the PVDF filters were blocked with 5\% non-fat milk for $1 \mathrm{~h}$ at room temperature (RT), the membranes were incubated with primary antibodies overnight at $4^{\circ} \mathrm{C}$. The primary antibodies used in this study were antiDYKDDDDK monoclonal antibody (diluted to 1:1000) and anti-KDEL monoclonal antibody (1:200). After the primary incubation, the filters were incubated with peroxidase-conjugated anti-mouse or anti-goat $\operatorname{IgG}$ secondary antibody (Jackson ImmunoRes. Lab Inc., West Grove, PA, USA; 1:10,000) for $1 \mathrm{~h}$ at RT. Immunoreactive bands were visualized with an ECL kit according to the manufacturer's directions (GE Healthcare, Tokyo). Densities of immunoreactive bands were measured using a luminescent image analyzer (LAS1000-plus; Fuji Photo, Tokyo).

\section{$R T-P C R$}

Total RNA was extracted from FLAG-SERT-transfected COS-7 cells using an RNAqueous Kit (Ambion, Life Technologies Japan) according to the manufacturer's protocol. After DNase treatment for $30 \mathrm{~min}$ at $37^{\circ} \mathrm{C}$, single-strand cDNA was synthesized from extracted RNA using the Super Script First-Strand Synthesis System for RT-PCR (Life Technologies Japan) according to the manufacturer's protocol. The resulting cDNA was then used as a template for typical PCR reactions. The primer set used to detect FLAG-SERT mRNA was 5'-GCAGATGGCATAACCAATCCC-3' and 5'-CTC GAGTTTACCACTCCCTAT-3'. The primer set used to detect GAPDH mRNA, an internal control, was 5'-AAGGTCGGTGTGAACGGATTTGG-3' and 5'-AGG GGCGGAGATGATGACCCTTTT-3'. The PCR conditions were as follows: 1 cycle of $2 \mathrm{~min}$ denaturing at $95^{\circ} \mathrm{C}, 25$ cycles of $30 \mathrm{~s}$ denaturing at $94^{\circ} \mathrm{C}, 90 \mathrm{~s}$ annealing at $60^{\circ} \mathrm{C}$, and 2 min extension at $72^{\circ} \mathrm{C}$.

\section{Observation of translocation of HT-fused SERT}

HT-fused protein can covalently bind to HT ligands, which were provided by Promega. To express FLAGSERT-HT, pCMV-tTA and pTetOp-FLAG-SERT-HT were transfected into COS-7 cells by electroporation, and transfected cells were then cultured in glass bottom dishes (3.5 cm in diameter, MatTek Corp.) for the appropriate amount of time. To stain FLAG-SERTHT with green or red fluorescence, the cells were incubated with culture medium containing Oregon greenconjugated HT ligand or TMR-HT ligand. To observe the lysosome, the cells were also incubated with $50 \mathrm{nM}$
LysoTracker red (Life Technologies Japan). Stained FLAG-SERT-HT-expressing cells were observed by confocal laser scanning fluorescence microscopy (LSM 510 inverted; Carl Zeiss, Jena, Germany). Time-lapse imaging of FLAG-SERT-HT was performed using a fluorescence microscope (BZ900; Keyence, Osaka) that contained a cell culture incubation apparatus (Stage Top Incubator, INU-K1-F1; TOKAI HIT, Fujinomiya).

\section{Immunocytochemistry and analysis of plasma membrane expression of SERT}

Immunocytochemistry was carried out for FLAGSERT-expressing HEK293 cells as previously described $(18,32)$. In brief, the cells were washed in $1 \mathrm{ml}$ PBS and fixed with $4 \%$ paraformaldehyde and $0.2 \%$ picric acid in $0.1 \mathrm{M}$ phosphate-buffered saline (PBS, pH 7.4) for $30 \mathrm{~min}$ at RT, washed twice with $1 \mathrm{ml} \mathrm{PBS}$, and then permeabilized with $500 \mu \mathrm{l}$ PBS supplemented with $0.3 \%$ Triton X-100 and 5\% normal goat serum (NGS) for 15 min. Then, the cells were washed twice in $1 \mathrm{ml}$ PBS with $0.03 \%$ Triton-X (PBS-T) and incubated for $<1 \mathrm{~h}$ at RT with the desired primary antibodies and for $<1 \mathrm{~h}$ at RT with the secondary antibodies in PBS-T and 5\% NGS. The primary and secondary antibody used was antiDYKDDDDK tag mouse monoclonal antibody (diluted 1:1000) and anti-mouse IgG conjugated with Alexa Fluor 488 (1:500), respectively.

Fluorescent signals were observed by confocal laser scanning fluorescent microscopy (LSM 510 invert). The intensity of fluorescence was measured using software associated with the confocal laser microscope as described previously (36).

\section{Statistical analyses}

Data analysis and statistics were performed using Prism 4 software (GraphPad Software, San Diego, CA, USA). Statistical significance was determined by Student's $t$-tests or one-way ANOVA followed by Dunnett's post test. If the $P$-value was less than 0.05 $(P<0.05)$, the difference was considered statistically significant.

\section{Results}

One-day treatment with 4-PBA increased the SERT uptake activity in FLAG-SERT-transfected COS-7 cells in a dose-dependent manner

pFLAG-SERT was transfected into COS-7 cells by electroporation. Twenty-four hours later, the cells were treated with various concentrations of 4-PBA. After an additional $24 \mathrm{~h}$ (48 $\mathrm{h}$ after transfection), the $\left[{ }^{3} \mathrm{H}\right] 5-\mathrm{HT}$ uptake assay was performed. The SERT uptake activity was defined as the amount of $\left[{ }^{3} \mathrm{H}\right] 5$-HT uptake per mg 


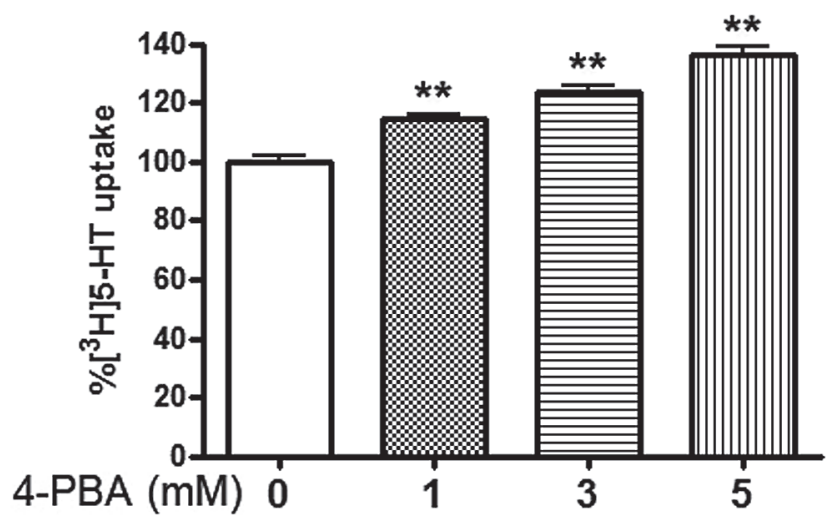

Fig. 1. Effects of 4-PBA on the 5-HT uptake activity of FLAGSERT-transfected COS-7 cells. pFLAG-SERT was transfected into COS-7 cells by electroporation. A $\left[{ }^{3} \mathrm{H}\right] 5$-HT uptake assay was performed $48 \mathrm{~h}$ after transfection. Treatment with 4-PBA was started 24 $h$ after transfection. Each column shows the percent $\left[{ }^{3} \mathrm{H}\right] 5$-HT uptake of non-treated control cells $(0 \mathrm{mM}$ 4-PBA). Data represent the mean \pm S.E.M. $(\mathrm{n}=8)(* * P<0.01$ vs. non-treated control, one-way ANOVA with Dunnett's post test).

of protein. As shown in Fig. 1, 4-PBA increased the 5-HT uptake in FLAG-SERT-expressed cells in a dosedependent manner.

4-PBA increased maturely glycosylated SERT and decreased immaturely glycosylated SERT without affecting the expression of FLAG-SERT $m R N A$

To elucidate the mechanism of how 4-PBA increased SERT uptake activity, the mRNA and protein levels of FLAG-SERT were examined. Total RNA was extracted from FLAG-SERT-transfected cells $48 \mathrm{~h}$ after treatment with $3 \mathrm{mM}$ 4-PBA. Extracted total RNA was then used as a template for RT-PCR. For semi-quantitative analysis, the number of PCR cycles was varied from 21 to 25. As shown in Fig. 2A, the amount of GAPDH PCR product increased in a cycle-dependent manner. The amount of SERT PCR products did not differ between 4-PBA-treated cells and control cells, which indicates that 4-PBA did not affect the transcription of FLAG-SERT mRNA in FLAG-SERT-transfected cells.

Western blot analysis was performed $48 \mathrm{~h}$ after transfection. As shown in Fig. 2B, the FLAG-SERT protein was recognized as multiple bands with molecular sizes of approximately 63,80 , and $120 \mathrm{kDa}$. Our previous study (37) clarified that a band with a molecular size of approximately $80 \mathrm{kDa}$ (arrow) corresponds to maturely glycosylated SERT, which should be functionally expressed at the plasma membrane. A band with a molecular size of approximately $63 \mathrm{kDa}$ (arrowhead) was identified as the form of SERT that is modified by high-mannose-type oligosaccharides, which is the immaturely glycosylated SERT that is retained in the ER. Our previous study also demonstrated that bands with molecular sizes greater than $80 \mathrm{kDa}$ correspond to oligomers of immaturely glycosylated SERT $(18,37)$. As shown in Fig. 2B, 24-h treatment with 4-PBA increased the amount of maturely glycosylated SERT (arrow) in a dose-dependent manner. Accordingly, 4-PBA decreased the amount of immaturely glycosylated SERT (arrowhead), which indicates that 4-PBA promotes its trafficking to the plasma membrane. As shown in Fig. $2 \mathrm{C}$, the ratio of the approximately $80-\mathrm{kDa}$ band densities (arrow) to approximately $63-\mathrm{kDa}$ ones (arrowhead) was significantly increased by the 4-PBA treatment. These findings suggest that 4-PBA increased SERT uptake activity by accelerating its membrane trafficking, which then increased the expression of maturely glycosylated SERT at the plasma membrane.

Butyrate, a histone deacetylase inhibitor, did not affect the expression pattern of SERT protein

Butyrate, an analogue of 4-PBA, lacks the phenyl group found on 4-PBA. Although both 4-PBA and butyrate have been reported to function as histone deacetylase inhibitors to a similar extent $(26,38,39)$, only 4 -PBA, and not butyrate, was observed to display chaperone activity (26). To further elucidate whether 4-PBA exerts its effects on SERT protein expression (Fig. 2B) via its chaperone activity, we compared the effects of 4-PBA and butyrate on SERT protein expression. Western blotting was performed after treating FLAG-SERTexpressing COS-7 cells with 4-PBA or butyrate for $24 \mathrm{~h}$. As shown in Fig. 3, A and B, 4-PBA again significantly increased maturely glycosylated SERT (arrow) and decreased immaturely glycosylated SERT (arrowhead) in a dose-dependent manner. In contrast, butyrate did not affect the expression pattern of SERT protein (Fig. 3: A and C). These results suggest that the specific action of 4-PBA on SERT protein expression is not mediated via inhibition of histone deacetylase, but is most likely mediated via its chaperone activity.

4-PBA upregulated SERT uptake activity even when de novo synthesis of FLAG-SERT was arrested

To further elucidate the mechanism underlying 4-PBA-induced up-regulation of SERT, we used COS-7 cells expressing FLAG-SERT in a tet-regulated manner. As mentioned in the Materials and Methods section, the addition of tetracycline to these cells can arrest the expression of SERT mRNA and protein. Therefore, using the tet-off system, we can selectively analyze the effects of 4-PBA on pre-existing SERT without influencing newly synthesized SERT. COS-7 cells were infected with two adenovirus vectors, Ad-CMV-tTA and TetOp- 

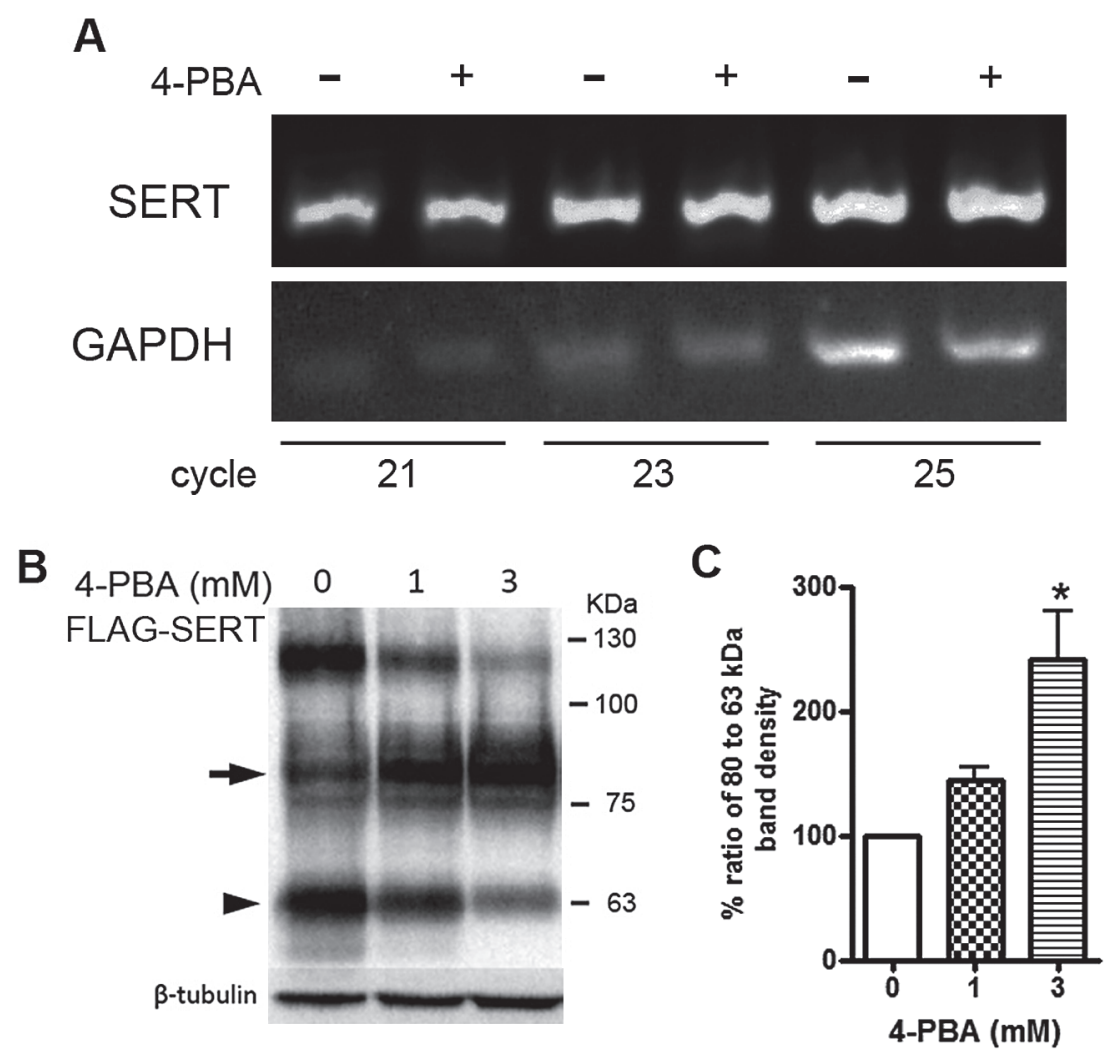

Fig. 2. Effects of 4-PBA on mRNA and protein expression of FLAG-SERT in FLAG-SERT-transfected COS-7 Cells. A: Semiquantitative RT-PCR analysis of FLAG-SERT mRNA. pFLAG-SERT was transfected into COS-7 cells by electroporation. Treatment with 4-PBA was started $24 \mathrm{~h}$ after transfection. Total RNA was extracted $48 \mathrm{~h}$ after transfection and used as a template for RT-PCR. RT-PCR was performed using a specific primer set for either FLAG-SERT or the internal control GAPDH. PCR products were loaded onto a $3.5 \%$ agarose gel containing EtBr and visualized with a transilluminator. Semi-quantitative RT-PCR analysis was performed by varying the number of PCR cycles. B: Effects of 4-PBA on the expression pattern of FLAG-SERT protein. The experimental time schedule is similar to that shown in Fig. 1. Western blot analysis was performed using antiDYKDDDDK (anti-FLAG) or anti- $\beta$-tubulin antibodies. Representative data are shown from 4 independent studies. The arrow indicates maturely glycosylated SERT, and the arrowhead indicates immaturely glycosylated SERT. C: Percent ratio of densities of the approximately $80-\mathrm{kDa}$ (maturely glycosylated SERT) band to the approximately $63-\mathrm{kDa}$ (immaturely glycosylated SERT) band. Each band density of SERT was normalized by that of $\beta$-tubulin. In each experiment, the ratio of the non-treated control was set at $100 \%$. Data represent the mean \pm the S.E.M. $(\mathrm{n}=3, * P<0.01$ vs. non-treated control, one-way ANOVA with Dunnett's post test).

FLAG-SERT. Twenty-four hours later, tetracycline $(1 \mu \mathrm{g} / \mathrm{ml})$ was simultaneously added with 4-PBA $(3 \mathrm{mM})$. RT-PCR analysis confirmed that the addition of tetracycline almost abolished mRNA expression of FLAGSERT (Fig. 4A). The $\left[{ }^{3} \mathrm{H}\right] 5$-HT uptake assay was performed 24 and $48 \mathrm{~h}$ after the start of drug application. As shown in Fig. 4, in the absence of 4-PBA, the 5-HT uptake was reduced by $30 \%$ and $40 \%$ in cells treated with tetracycline $(1 \mathrm{mg} / \mathrm{ml})$ for 24 and $48 \mathrm{~h}$, respectively. However, 4-PBA still significantly increased SERT uptake activity even when de novo synthesis of SERT was stopped by the tet-off system, which suggests that 4-PBA affects pre-existing SERT protein by a posttranslational mechanism.
4-PBA restored the uptake activity of the C-terminaldeleted mutant SERT (SERTACT) and relieved ER stress induced by SERTACT

The C-terminus of SERT is known to play an important role in SERT membrane trafficking $(17,18)$. We previously reported that SERT $\triangle \mathrm{CT}$, which has a dramatically reduced uptake activity, was retained in ER and induced ER stress (18). To elucidate whether 4-PBA improves membrane trafficking of SERT $\triangle \mathrm{CT}$, wild-type FLAG-SERT or FLAG-SERT $\triangle \mathrm{CT}$ was transfected into COS-7 cells. Experiments were performed using the same time schedule as that shown in Fig. 1. As shown in Fig. 5A, and as we previously reported (18), the uptake activity of SERT $\triangle \mathrm{CT}$ was reduced to almost $5 \%$ of that 

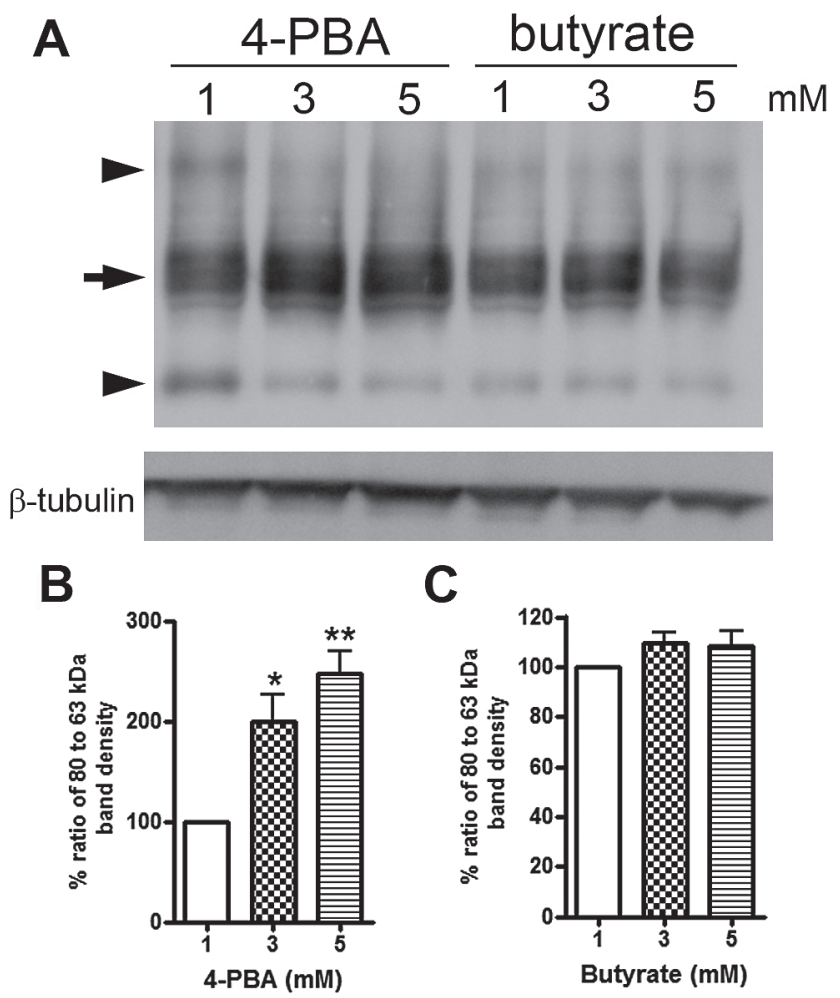

Fig. 3. Western blot analysis of SERT protein expression in 4-PBAor butyrate-treated COS-7 cells. A: Comparison of effects of 4-PBA or butyrate on SERT protein expression. The experimental schedule and procedure were similar to that in Figs. 1 and 2B. Western blot analysis was performed using samples prepared from 4-PBA $(1,3$, and $5 \mathrm{mM})$ - or butyrate $(1,3$, and $5 \mathrm{mM})$-treated cells. The arrow and arrowhead indicate maturely glycosylated SERT and immaturely glycosylated SERT, respectively. B and C: Percent ratio of densities of the approximately $80-\mathrm{kDa}$ (maturely glycosylated SERT) band to the approximately $63-\mathrm{kDa}$ (immaturely glycosylated SERT) band in 4-PBA treated and butyrate-treated cells, respectively. Each band density of SERT was normalized by that of $\beta$-tubulin. In each experiment, the ratio of $1 \mathrm{mM} 4-\mathrm{PBA}$-treated cells was set at $100 \%$. Data represent the mean \pm the S.E.M. $\left(\mathrm{n}=4,{ }^{*} P<0.05,{ }^{* *} P<0.01\right.$ vs. $1 \mathrm{mM}$ 4-PBA-treated group, one-way ANOVA with Dunnett's post test).

of wild-type SERT. Treatment with 4-PBA (3 mM) upregulated both wild-type SERT activity and SERT $\triangle \mathrm{CT}$ activity (Fig. 5B). In addition, 4-PBA increased the uptake activity of SERT $\triangle \mathrm{CT}$ to a greater extent than wild-type SERT (Fig. 5B). We examined how 4-PBA affects both the expression pattern of SERT protein and ER stress. As shown in Fig. 5C, maturely glycosylated SERT was rarely observed in SERT $\triangle \mathrm{CT}$-expressing cells compared with wild-type SERT (Fig. 2B). 4-PBA significantly decreased the level of immaturely glycosylated SERT in a dose-dependent manner (Fig. 5: C and D). As shown in Fig. 5E, the anti-KDEL antibody recognized GRP94 and GRP78, which are marker proteins of ERstress induction. 4-PBA decreased the levels of these

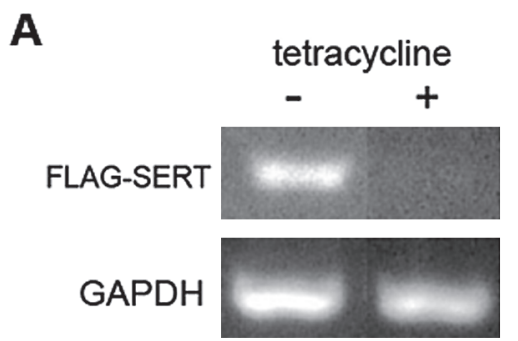

B

\section{4 hours-treatment}

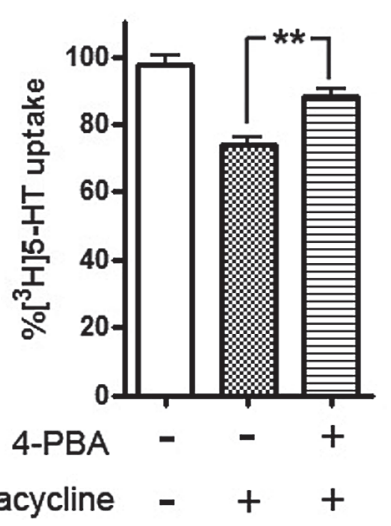

48 hours-treatment

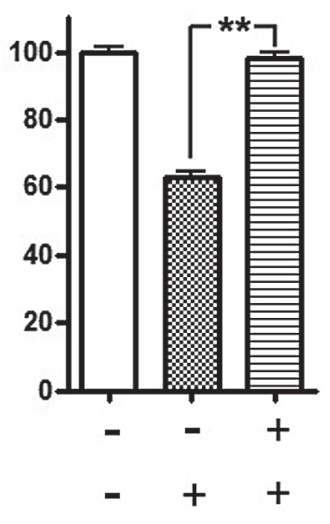

Fig. 4. Effects of 4-PBA on the uptake activity and expression of SERT under the condition in which de novo synthesis of SERT was arrested by the tet-off system. COS-7 cells were infected with two adenoviral vectors, Ad-CMV-tTA and Ad-TetOp-FLAG-SERT. Twenty-four hours after transfection, tetracycline $(1 \mathrm{mg} / \mathrm{ml})$ and 4-PBA (3 mM) were simultaneously applied. A: Arrest of FLAGSERT expression by the addition of tetracycline. RT-PCR was performed using total RNA that was extracted from cells treated with tetracycline for $24 \mathrm{~h}$ as templates. B: Effects of 4-PBA on the SERT uptake activity under the condition of tet-off system. After a 24- or 48-hour treatment with $3 \mathrm{mM} 4$-PBA and tetracycline, a $\left.{ }^{3} \mathrm{H}\right] 5-\mathrm{HT}$ uptake assay was performed. Each column shows the percent $\left[{ }^{3} \mathrm{H}\right] 5-$ HT uptake of non-treated control cells. Data represent the mean \pm S.E.M. $(\mathrm{n}=8)\left({ }^{*} P<0.01\right.$, vs. tetracycline-treated and 4-PBAnon-treated cells, one-way ANOVA with Dunnett's post test).

marker proteins in SERTACT-expressed COS-7 cells in a dose-dependent manner (Fig. 5E). These effects of 4-PBA on GRP78 expression was statistically significant (Fig. 5F). These findings suggest that 4-PBA functions as a chaperone and thereby decreases immaturely glycosylated SERTACT stacked in the ER and that 4-PBA thus relieves ER stress.

\section{Imaging of SERT-HaloTag (SERT-HT) translocation}

Receptors and transporters, including SERT, are folded into a trans-membrane conformation in the ER after they are translated, and they are then trafficked to the plasma membrane via the Golgi apparatus. Thereafter, the proteins are translocated into the lysosome via endo- 
A

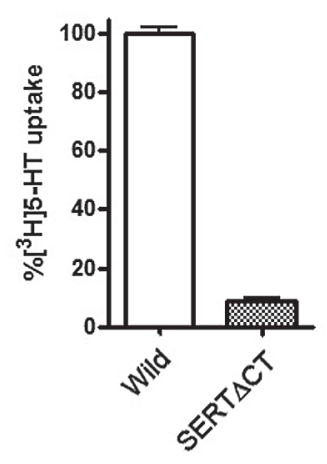

C

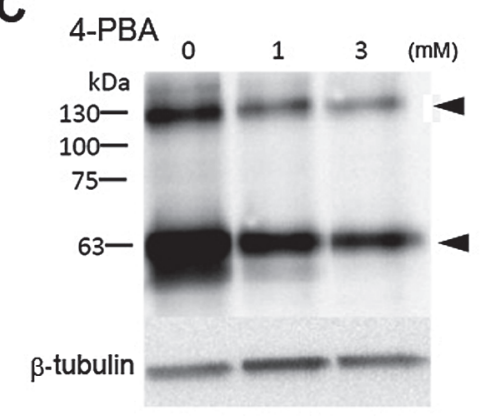

$\mathbf{E}$

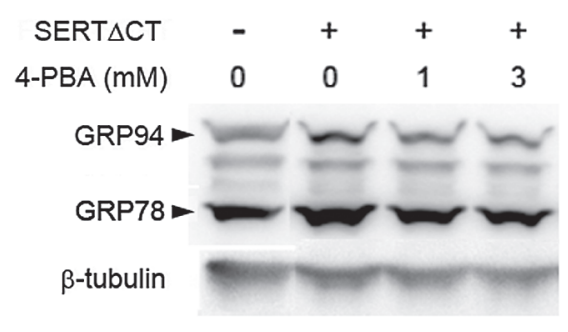

B

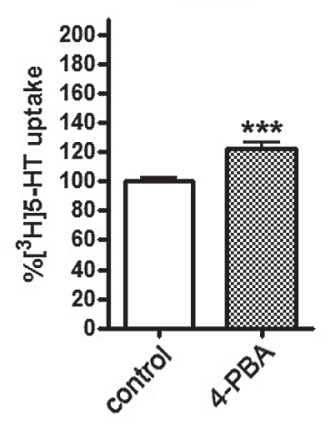

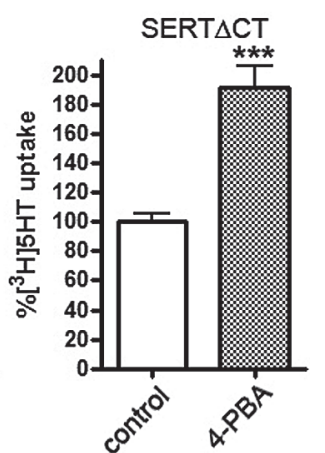

D

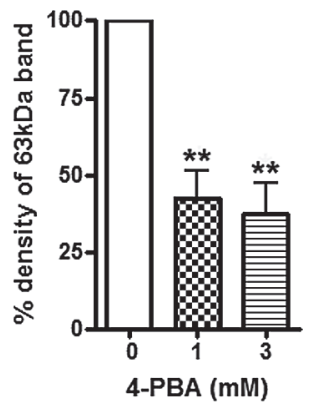

$\mathbf{F}$

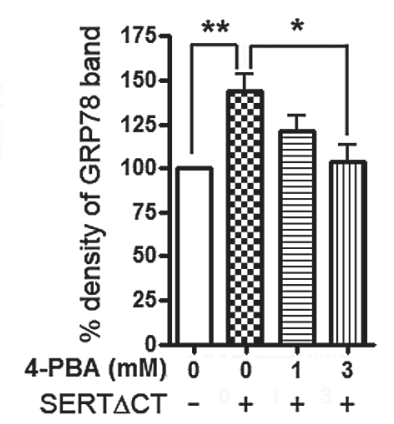

Fig. 5. Effects of 4-PBA on the protein expression and uptake activity of SERT $\triangle \mathrm{CT}$. A: Comparison of 5-HT uptake activity between wild-type SERT- and SERT $\triangle$ CT-transfected cells. pFLAG-SERT or pFLAG-SERT $\triangle \mathrm{CT}$ was transfected into COS-7 cells. A $\left[{ }^{3} \mathrm{H}\right] 5$-HT uptake assay was performed $48 \mathrm{~h}$ after transfection. Each column shows the percent of $\left[{ }^{3} \mathrm{H}\right] 5$-HT uptake of wild-type SERT-expressing cells. Data represent the mean \pm S.E.M. $(n=8)$. B: Effects of 4-PBA $(3 \mathrm{mM})$ on the uptake activity of wild-type SERT and SERT $\triangle \mathrm{CT}$. Each column shows the percent of $\left[{ }^{3} \mathrm{H}\right] 5$-HT uptake of non-treated control cells. Data represent the mean \pm S.E.M. $(\mathrm{n}=8)$. $(* * * P<0.001$ vs. non-treated controls, Student's $t$-test). C: Effects of 4-PBA $(3 \mathrm{mM})$ on the expression pattern of FLAG-SERT protein. The experimental schedule was similar to that in Fig. 2B. Western blot analysis of FLAGSERT $\triangle C T$ was performed $48 \mathrm{~h}$ after transfection using an anti-DYKDDDDK (anti-FLAG) antibody. Arrowheads indicate immaturely glycosylated SERT $\triangle$ CT. Maturely glycosylated SERTDCT was not observed. Representative immunoblotting data from three independent studies are shown. D: Quantitative analysis of the expression level of approximately 63-kDa immaturely glycosylated SERT $\triangle \mathrm{CT}$. Each band density of SERT $\triangle \mathrm{CT}$ was normalized by that of $\beta$-tubulin. In each experiment, the density of SERT $\triangle C T$ in the non-treated control was set at $100 \%$. Data represent the mean \pm the S.E.M. $(\mathrm{n}=8, * * P<0.01$, vs. non-transfected control, one-way ANOVA with Dunnett's post test). E: Effects of 4-PBA on the expression of ER stress markers, GRP94 and GRP78. Experimental schedule was similar to that in Fig. 2B. Western blot analysis for GRP94 and GRP78 was performed using an anti-KDEL antibody. Representative immunoblotting data from four independent studies are shown. F: Quantitative analysis of the expression level of GRP78. Each band density of GRP78 was normalized by that of $\beta$-tubulin. In each experiment, the density of GRP78 in non-transfected control was set at $100 \%$. Data represent the mean \pm the S.E.M. $(\mathrm{n}=4, * * P<0.01, * P<0.05$ vs. non-treated and SERT-transfected cells, one-way ANOVA with Dunnett's post test). 
cytosis. To observe the time-lapse translocation of SERT, pCMV-tTA and pTetOp-FLAG-SERT-HT were simultaneously transfected into COS-7 cells by electroporation, and the localization of SERT was observed using confocal laser scanning microscopy. Prior to the imaging research, we examined weather FLAG-SERT-HT has the uptake activity of 5-HT. We performed the kinetic analysis of FLAG-SERT-HT-expressing COS-7 cells by varying the concentration of 5-HT from 0.1 to $20 \mu \mathrm{M}$. Results revealed that the 5-HT uptake activity with $\mathrm{Km}$ value of $3.9 \mu \mathrm{M}$ was saturated at the 5-HT concentration of more than $10 \mu \mathrm{M}$, indicating that FLAG-SERT-HT is functional (data not shown). First, we attempted to confirm whether expressed FLAG-SERT-HT could accurately report membrane trafficking. Thus, cells were stained with Oregon green-conjugated HT (green signal) ligand and LysoTracker (red signal) 1 day after transfection to evaluate the localization of both FLAG-SERTHT and lysosomes, respectively. To eliminate the possibility that newly synthesized FLAG-SERT-HT proteins influence the observation of pre-existing FLAG-SERTHT, tetracycline $(1 \mathrm{mg} / \mathrm{ml})$ was added after staining to arrest the de novo synthesis of FLAG-SERT-HT. As shown in Fig. 6A, 1 day after staining, SERT was expressed throughout the cytosol, including in an ERlike apparatus, whereas SERT was rarely co-localized with lysosomes (Fig. 6A, upper row). Two days after staining, the plasma membrane localization of SERT was increased (Fig. 6A, middle row). Three days after staining, SERT expression was decreased in the plasma membrane and it was translocated to dot-like intracellular apparatuses, which co-localized with lysosomes. These phenomena reflect the process of SERT membrane trafficking, which supports the validity of this method for observing SERT translocation in live cells.

We examined whether 4-PBA affected the localization of FLAG-SERT-HT expressed in COS-7 cells. Forty eight hours after the transfection, cells treated with $3 \mathrm{mM}$ 4-PBA for $24 \mathrm{~h}$ were stained with oregon greenconjugated HT ligand. As shown in Fig. 6B, FLAGSERT-HT localized at the cell edge (arrowhead) was increased by 4-PBA, but in contrast, its expression at the perinuclear region (arrow), which is supposed to be the ER or Golgi apparatus, was decreased.

In the Supplementary Fig. and movie (available only in the online version), we attempted a time-lapse observation of SERT translocation in live cells. Twenty-four hours after transfection, FLAG-SERT-HT-expressing cells were stained with TMR-conjugated HT ligand (red signal). Time-lapse imaging was then started immediately after treatment with $3 \mathrm{mM} 4-\mathrm{PBA}$ and tetracycline $(1 \mathrm{mg} / \mathrm{ml})$. In 4-PBA-treated cells, the signal of FLAG-SERT-HT at the plasma membrane gradually increased, especially in the regions indicated by the arrowheads, whereas accumulation of SERT in the plasma membrane was not observed in non-treated control cells (Supplementary Fig. and movie). In addition, perinuclear FLAG-SERT-HT was decreased in 4-PBA treated cells, in contrast to non-treated control cells showing slight increment of perinuclear FLAGSERT-HT. These results support the idea that 4-PBA accelerates membrane trafficking of SERT to the plasma membrane, thereby increasing the 5-HT uptake activity of SERT-transfected cells.

To further confirm the accelerating effects of 4-PBA on membrane trafficking of SERT, plasma membrane expression of FLAG-SERT was analyzed by fluorescent immunohistochemistry (36). For this purpose, we expressed FLAG-SERT in HEK293 cells because the cellular morphology and SERT expression pattern of HEK293 cells is more homogeneous than those of COS7 cells. Again, we examined whether 4-PBA up-regulates the 5-HT uptake activity in FLAG-SERT-expressing HEK293 cells. Experiments were performed using the same time schedule as that shown in Figs. 1 and 5. As shown in Fig. 7A, 4-PBA dose-dependently increased 5-HT uptake activity in FLAG-SERT-expressing HEK293 cells as well as COS-7 cells (Fig. 7A). The localization of FLAG-SERT in the expressed HEK293 cells was visualized by fluorescent immunohistochemistry using anti-DYKDDDDK antibody. Figure 7B shows the representative stained cells and line-profiling of fluorescent intensity that did not transverse the nucleus as indicated by the line. Quantification of SERT plasma membrane expression was performed as previously reported (36). Briefly, the green-colored area corresponds to the summation of the fluorescent intensity in the plasma membrane. The green-colored arrow corresponds to the length of the cell membrane. We calculated the average fluorescent intensity in the plasma membrane as the ratio of the green area to the length of the green arrow. Similarly, we calculated the average fluorescent intensity in the cytosol using the orange-colored area and arrow. Finally, we calculated the ratio of the average fluorescent intensity in the plasma membrane to the average fluorescent intensity in the cytosol as an index that indicates the plasma membrane expression of FLAG-SERT. Representative data showed that 4-PBA increased the plasma membrane staining and decreased the cytosolic staining of FLAG-SERT (Fig. 7C). Quantitative analysis revealed that the index of SERT plasma membrane expression was significantly higher in $3 \mathrm{mM}$ 4-PBAtreated cells than in non-treated control cells (Fig. 7D). 
A

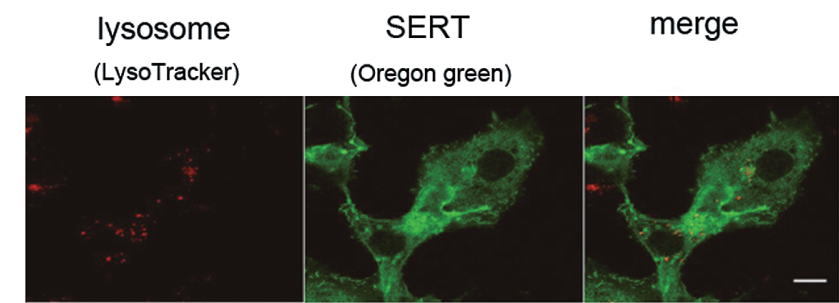

Day 1

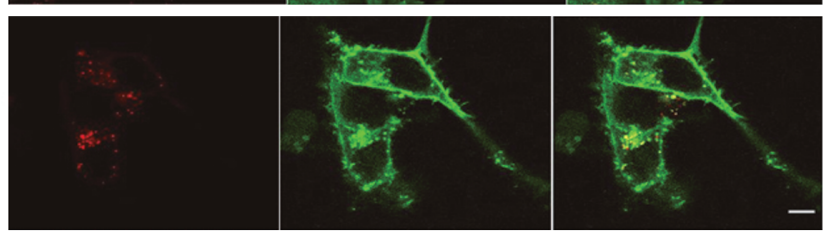

Day 2

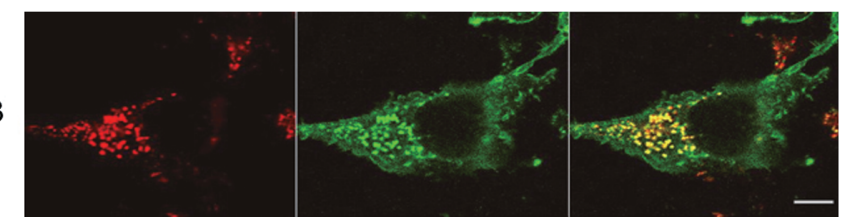

B

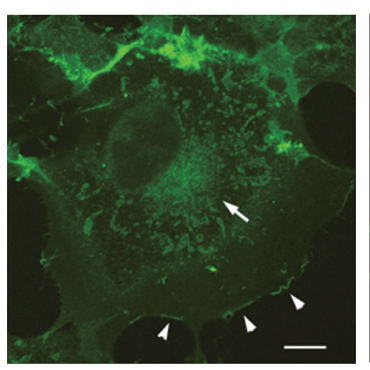

4-PBA (3 mM)

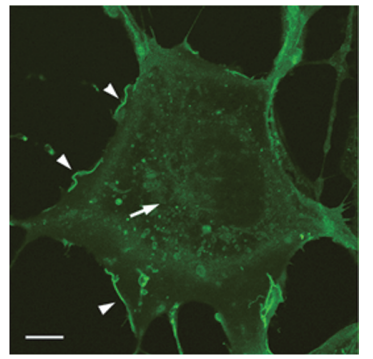

Fig. 6. Effects of 4-PBA on SERT translocation. A: Time-course study of SERT-HT translocation. COS-7 cells were transfected with a plasmid encoding CMV-tTA or TetOp-FLAG-SERT-HT, and the localization of SERT was observed using confocal laser scanning microscopy. SERT-HT was visualized by Oregon green-conjugated HaloTag ligand (green signal) one day after transfection. Lysosomes were visualized using LysoTracker (red signal). Tetracycline $(1 \mathrm{mg} / \mathrm{ml})$ was added to eliminate the influence of newly synthesized SERT-HT. Translocation of SERT-HT was followed for 3 days after staining. Bars indicate $10 \mu \mathrm{m}$. B: Comparison of SERT-HT localization between non-treated control and 4-PBA (3 mM)-treated cells. Treatment with 4-PBA was started $24 \mathrm{~h}$ after transfection. SERT-HT was stained with Oregon green-conjugated HaloTag ligand $48 \mathrm{~h}$ after transfection. Arrowheads indicate accumulation of SERT-HT at the cell edge. Arrows indicate SERT-HT at the perinuclear region. Bars indicate $10 \mu \mathrm{m}$.

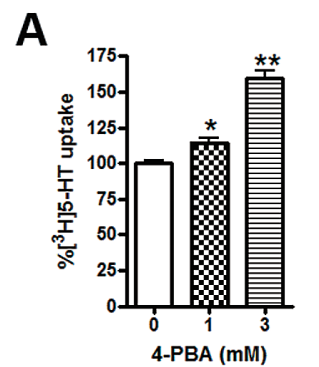

C

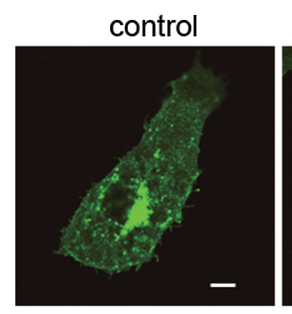

B

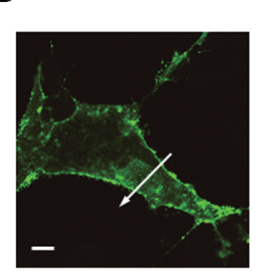

4-PBA (3 mM)

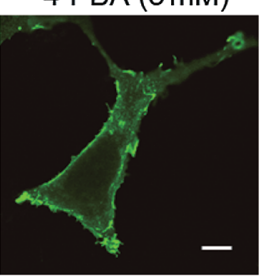

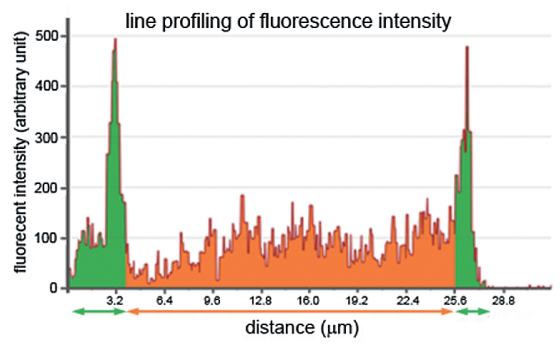

D

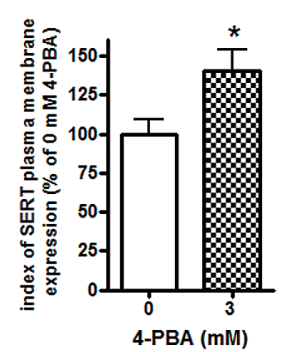

Fig. 7. Quantitative analysis of plasma membrane expression of SERT in FLAG-SERT-expressing HEK293 cells. A: Effects of 4-PBA on the 5-HT uptake activity in FLAG-SERT-expressing HEK 293 cells. pFLAG-SERT was transfected into HEK293 cells by electroporation. A [ $\left.{ }^{3} \mathrm{H}\right] 5-\mathrm{HT}$ uptake assay was performed $48 \mathrm{~h}$ after transfection. Treatment with 4-PBA was started $24 \mathrm{~h}$ after transfection. Each column shows the percent $\left[{ }^{3} \mathrm{H}\right] 5-\mathrm{HT}$ uptake of non-treated control cells $(0 \mathrm{mM}$ 4-PBA). Data represent the mean \pm S.E.M. $(\mathrm{n}=4)(* P<0.05, * * P<0.01$, vs. non-treated control, one-way ANOVA with Dunnett's post test). B: Line profiling of fluorescence intensity. The experimental schedule was similar to that in Fig. 7A. Cells were immunostained using anti-DYKDDDDK (anti-FLAG) antibody and developed with Alexa 488-labeled secondary antibody. Images were obtained by a confocal laser scanning microscope. Fluorescence intensity along the line, as shown in the left panel, was measured using the computer software associated with the confocal laser scanning microscope. The green and orange colored areas indicate the summation of fluorescent intensity in the plasma membrane and cytosol, respectively. The green and orange colored arrows indicate the length of the plasma membrane and cytosol, respectively. A plasma membrane expression index was calculated as described in the results. C: Comparison of FLAG-SERT localization between nontreated control and 4-PBA (3 mM)-treated cells. Representative immunostained control and 4-PBA-treated cells are shown. D: Quantitative analysis of plasma membrane expression of SERT in non-treated control and 4-PBA-treated cells. Each column shows the percent index of non-treated control cells $\left(0 \mathrm{mM}\right.$ 4-PBA). Data represent the mean \pm S.E.M. $\left(\mathrm{n}=19\right.$ and 17 in control and 4-PBA-treated cells, respectively; ${ }^{*} P<0.05$, vs. non-treated control, Student's $t$-test). 


\section{Discussion}

There has been a focus on chemical chaperones as potential therapeutic drugs to improve ER stress-related pathology $(22-24)$. The aim of this study was to elucidate whether these chemical chaperones have the potential to affect serotonergic neural transmission by accelerating membrane trafficking of SERT. If this is the case, these drugs could provide a novel therapeutic approach in the treatment of SERT-related disorders.

In this study, we focused on the chemical chaperone 4-PBA. 4-PBA, a carboxylic acid with a phenyl group and 4 carbon chains, has a variety of actions, including enhancing transcription by inhibiting histone deacetylase, inhibiting protein accumulation, and accelerating trafficking of unfolded proteins to the plasma membrane (26).

One-day treatment with 4-PBA increased SERT activity in a dose-dependent manner (Fig. 1) by increasing maturely glycosylated SERT and decreasing immaturely glycosylated SERT (Fig. 2: B and C). Our previous studies demonstrated that maturely glycosylated SERT, which is localized to the plasma membrane, was a functional transporter, while immaturely glycosylated SERT was retained in the ER and was not functional $(18,37)$. Taken together, these findings suggest that 4-PBA exerts its action on SERT activity by functioning as a chemical chaperone and thereby accelerates the membrane trafficking of SERT.

We performed several experiments to exclude the possibility that 4-PBA affects SERT activity by functioning as an inhibitor of histone deacetylase. RT-PCR analysis revealed that the transcription of FLAG-SERT from transfected vectors was not changed (Fig. 2A). In addition, we used western blot analysis to compare the influence of butyrate on the expression patterns of SERT proteins with that of 4-PBA. Butyrate is an analogue of 4-PBA and has the same potential to act as a histone deacetylase inhibitor as 4-PBA, but it does not act as a chemical chaperone $(26,38,39)$. In contrast to 4-BPA, butyrate did not affect the expression patterns of SERT proteins (Fig. 3: A and B). Furthermore, we examined the effects of 4-PBA on SERT activity in cells in which de novo synthesis of SERT was abolished by the tet-off system (Fig. 4A). As shown in Fig. 4B, 4-PBA also increased SERT activity under this condition, which indicates that 4-PBA acts on pre-existing SERT in a posttranslational manner. These results also support the idea that 4-PBA enhances membrane trafficking of SERT by functioning as a chemical chaperone.

We previously demonstrated that the SERT $\triangle \mathrm{CT}$, which was almost fully retained in the ER and induced ER stress, had very low levels of 5-HT uptake activity when expressed in COS-7 cells (18). To further elucidate the effects of 4-PBA on SERT regulation, we attempted to examine whether 4-PBA restored the function of SERT $\triangle \mathrm{CT}$ by functioning as a chemical chaperone. As shown in Fig. 5B, 5-HT uptake via SERT $4 \mathrm{CT}$ was also significantly increased by 4-PBA, which is similar to that observed with wild-type SERT. The rate of increase was higher in SERT $\triangle \mathrm{CT}$ than in wild-type SERT. Western blot analysis of the SERT $\triangle \mathrm{CT}$ protein showed that 4-PBA reduced the level of immaturely glycosylated SERT in a dose-dependent manner, although an increase in the level of maturely glycosylated SERT was not detected (Fig. 5: C and D). It is unknown why maturely glycosylated SERT was not observed instead of the observed decrease in immaturely glycosylated SERT. The expression of maturely glycosylated SERT may be lower than the level detectable by western blotting. Alternatively, in the case of SERT $\triangle \mathrm{CT}$, immaturely glycosylated SERT may be trafficked to the plasma membrane and may behave as a functional transporter. Additionally, in this study, the total expression level of SERT $\triangle \mathrm{CT}$ was decreased by 4-PBA (Fig. 4C), which suggests that the chemical chaperone activity of 4-PBA relieved the SERT $\triangle \mathrm{CT}$ stacked in the ER and promoted its degradation via the ERAD (endoplasmic reticulumassociated degradation) system (40), thereby reducing ER stress. Indeed, in SERT $\triangle \mathrm{CT}$-expressing cells, 4-PBA reduced the levels of ER stress marker proteins GRP94 and GRP78, which are endogenous chaperones (Fig. 5: $\mathrm{E}$ and $\mathrm{F}$ ). These findings further support the idea that 4-PBA acts on ER-stacked immaturely glycosylated SERT as a chemical chaperone and promotes its membrane trafficking or degradation via the ERAD system.

To visualize the effects of 4-PBA on SERT localization, we expressed SERT-HT and visualized it by using a fluorescence-conjugated HT ligand. By the combined use of the HT and tet-off systems, we can selectively follow the SERT stained by the HT ligand for long periods of time without detecting the translocation of de novo synthesized SERT. Lysosome co-localization experiments revealed that this system is suitable for the time-lapse observation of SERT translocation (Fig. 6A). The SERT-HT localized at the cell edge was more prominent in 4-PBA-treated cells than in non-treated control cells $24 \mathrm{~h}$ after the treatment (Fig. 6B). In time-lapse imaging of SERT-HT translocation, SERT was gradually translocated to the plasma membrane after the application of 4-PBA (Supplementary Fig. and movie). Furthermore, the plasma membrane expression of FLAG-SERT in the expressed HEK293 cells was significantly increased by 4-PBA (Fig. 7: C and D). These results support the idea that 4-PBA accelerates SERT membrane trafficking. 
In brain ischemia, neural aging and neurodegeneration, ER stress is caused by peroxidative stress and the accumulation of proteins that are resistant to degradation $(19-21)$. In such pathological states, ER stress may induce the dysfunction of serotonergic neurotransmission by disturbing the membrane trafficking of SERT, which could result in the occurrence of a SERTrelated disorder, such as depression. Chemical chaperones, such as 4-PBA, may have the potential to improve these situations and may represent a novel approach for treating SERT-related diseases.

\section{Acknowledgments}

This study was supported by a Grant-in-Aid for Scientific Research from the Ministry of Education, Culture, Sports, Science and Technology, Japan and by grants from the Takeda Science Foundation, the Uehara Memorial Foundation, the Naito Foundation, the Suzuken Memorial Foundation, the Tokyo Biochemical Research Foundation, and the Japanese Smoking Research Association. This work was performed using equipment at the Analysis Center of Life Science, Hiroshima University.

\section{References}

1 Stahl SM. Essential Psychopharmacology: Neuroscientific Basis and Practical Applications. 2nd ed. Cambridge: Cambridge University Press; 2000.

2 Blakely RD, Berson HE, Fremeau RT Jr, Caron MG, Peek MM, Prince HK, et al. Cloning and expression of a functional serotonin transporter from rat brain. Nature. 1991;354:66-70.

3 Kuhar MJ, Roth RH, Aghajanian GK. Synaptosomes from forebrains of rats with midbrain raphe lesions: selective reduction of serotonin uptake. J Pharmacol Exp Ther. 1972;181: 36- 45.

4 Han DD, Gu HH. Comparison of the monoamine transporters from human and mouse in their sensitivities to psychostimulant drugs. BMC Pharmacol. 2006;6:6.

5 Olivier B, Soudijn W, van Wijngaarden I. Serotonin, dopamine and norepinephrine transporters in the central nervous system and their inhibitors. Prog Drug Res. 2000;54:59-119.

6 Rudnick G, Wall SC. Binding of the cocaine analog 2 beta-[3H] carboxymethoxy-3 beta-(4-fluorophenyl)tropane to the serotonin transporter. Mol Pharmacol. 1991;40:421-426.

7 Caspi A, Sugden K, Moffitt TE, Taylor A, Craig IW, Harrington $\mathrm{H}$, et al. Influence of life stress on depression: moderation by a polymorphism in the 5-HTT gene. Science. 2003;301:386-389.

8 Lesch KP, Bengel D, Heils A, Sabol SZ, Greenberg BD, Petri S, et al. Association of anxiety-related traits with a polymorphism in the serotonin transporter gene regulatory region. Science. 1996;274:1527-1531.

9 Ozaki N, Goldman D, Kaye WH, Plotnicov K, Greenberg BD, Lappalainen J, et al. Serotonin transporter missense mutation associated with a complex neuropsychiatric phenotype. Mol Psychiatry. 2003;8:933-936.

10 Prasad HC, Steiner JA, Sutcliffe JS, Blakely RD. Enhanced activity of human serotonin transporter variants associated with autism. Philos Trans R Soc Lond B Biol Sci. 2009;364:163-173.
11 Sutcliffe JS, Delahanty RJ, Prasad HC, McCauley JL, Han Q, Jiang L, et al. Allelic heterogeneity at the serotonin transporter locus (SLC6A4) confers susceptibility to autism and rigidcompulsive behaviors. Am J Hum Genet. 2005;77:265-279.

12 Tate CG, Blakely RD. The effect of N-linked glycosylation on activity of the $\mathrm{Na}(+)$ - and $\mathrm{Cl}(-)$-dependent serotonin transporter expressed using recombinant baculovirus in insect cells. J Biol Chem. 1994;269:26303-26310.

13 Samuvel DJ, Jayanthi LD, Bhat NR, Ramamoorthy S. A role for p38 mitogen-activated protein kinase in the regulation of the serotonin transporter: evidence for distinct cellular mechanisms involved in transporter surface expression. J Neurosci. 2005;25: 29-41.

14 Zhu CB, Carneiro AM, Dostmann WR, Hewlett WA, Blakely RD. p38 MAPK activation elevates serotonin transport activity via a trafficking-independent, protein phosphatase $2 \mathrm{~A}$-dependent process. J Biol Chem. 2005;280:15649-15658.

15 Zhu CB, Hewlett WA, Feoktistov I, Biaggioni I, Blakely RD. Adenosine receptor, protein kinase $\mathrm{G}$, and $\mathrm{p} 38$ mitogen-activated protein kinase-dependent up-regulation of serotonin transporters involves both transporter trafficking and activation. Mol Pharmacol. 2004;65:1462-1474.

16 El-Kasaby A, Just H, Malle E, Stolt-Bergner PC, Sitte HH, Freissmuth M, et al. Mutations in the carboxyl-terminal SEC24 binding motif of the serotonin transporter impair folding of the transporter. J Biol Chem. 2010;285:39201-39210.

17 Larsen MB, Fjorback AW, Wiborg O. The C-terminus is critical for the functional expression of the human serotonin transporter. Biochemistry. 2006;45:1331-1337.

18 Nobukuni M, Mochizuki H, Okada S, Kameyama N, Tanaka A, Yamamoto $\mathrm{H}$, et al. The $\mathrm{C}$-terminal region of serotonin transporter is important for its trafficking and glycosylation. J Pharmacol Sci. 2009;111:392-404.

19 Bossy-Wetzel E, Schwarzenbacher R, Lipton SA. Molecular pathways to neurodegeneration. Nat Med. 2004;10 Suppl:S2-S9.

20 Paschen W, Mengesdorf T. Cellular abnormalities linked to endoplasmic reticulum dysfunction in cerebrovascular diseasetherapeutic potential. Pharmacol Ther. 2005;108:362-375.

21 Paschen W, Mengesdorf T. Endoplasmic reticulum stress response and neurodegeneration. Cell Calcium. 2005;38:409-415.

22 Ozcan U, Yilmaz E, Ozcan L, Furuhashi M, Vaillancourt E, Smith RO, et al. Chemical chaperones reduce ER stress and restore glucose homeostasis in a mouse model of type 2 diabetes. Science. 2006;313:1137-1140.

23 Powell K, Zeitlin PL. Therapeutic approaches to repair defects in deltaF508 CFTR folding and cellular targeting. Adv Drug Deliv Rev. 2002;54:1395-1408.

24 Tamarappoo BK, Verkman AS. Defective aquaporin-2 trafficking in nephrogenic diabetes insipidus and correction by chemical chaperones. J Clin Invest. 1998;101:2257-2267.

25 Burrows JA, Willis LK, Perlmutter DH. Chemical chaperones mediate increased secretion of mutant alpha 1-antitrypsin (alpha 1-AT) Z: A potential pharmacological strategy for prevention of liver injury and emphysema in alpha 1-AT deficiency. Proc Natl Acad Sci U S A. 2000;97:1796-1801.

26 Kubota K, Niinuma Y, Kaneko M, Okuma Y, Sugai M, Omura T, et al. Suppressive effects of 4-phenylbutyrate on the aggregation of Pael receptors and endoplasmic reticulum stress. J Neurochem. 2006;97:1259-1268.

27 Rubenstein RC, Zeitlin PL. Sodium 4-phenylbutyrate down- 
regulates Hsc70: implications for intracellular trafficking of DeltaF508-CFTR. Am J Physiol Cell Physiol. 2000;278: C259-C267.

28 Wei H, Kim SJ, Zhang Z, Tsai PC, Wisniewski KE, Mukherjee $\mathrm{AB}$. ER and oxidative stresses are common mediators of apoptosis in both neurodegenerative and non-neurodegenerative lysosomal storage disorders and are alleviated by chemical chaperones. Hum Mol Genet. 2008;17:469-477.

29 Crowe JH. Trehalose as a "chemical chaperone": fact and fantasy. Adv Exp Med Biol. 2007;594:143-158.

30 Qi X, Hosoi T, Okuma Y, Kaneko M, Nomura Y. Sodium 4phenylbutyrate protects against cerebral ischemic injury. Mol Pharmacol. 2004;66:899-908.

31 Mochizuki H, Amano T, Seki T, Matsubayashi H, Mitsuhata C, Morita $\mathrm{K}$, et al. Role of $\mathrm{C}$-terminal region in the functional regulation of rat serotonin transporter (SERT). Neurochem Int. 2005;46:93-105.

32 Seki T, Shimahara T, Yamamoto K, Abe N, Amano T, Adachi N, et al. Mutant gammaPKC found in spinocerebellar ataxia type 14 induces aggregate-independent maldevelopment of dendrites in primary cultured Purkinje cells. Neurobiol Dis. 2009;33:260-273.

33 Yamamoto K, Seki T, Adachi N, Takahashi T, Tanaka S, Hide I, et al. Mutant protein kinase $\mathrm{C}$ gamma that causes spinocerebellar ataxia type 14 (SCA14) is selectively degraded by autophagy. Genes Cells. 2010;15:425-438.

34 Sakai N, Sasaki K, Nakashita M, Honda S, Ikegaki N, Saito N.
Modulation of serotonin transporter activity by a PKC activator and an inhibitor of type 1 and 2A serine/threonine phosphatases. J Neurochem. 1997;68:2618-2624.

35 Seki T, Adachi N, Ono Y, Mochizuki H, Hiramoto K, Amano T, et al. Mutant protein kinase Cgamma found in spinocerebellar ataxia type 14 is susceptible to aggregation and causes cell death. J Biol Chem. 2005;280:29096-29106.

36 Yammamoto H, Tanaka S, Tanaka A, Hide I, Seki T, Sakai N. Long-Term Exposure of RN46A Cells expressing serotonin transporter (SERT) to a cAMP analog up-regulates SERT activity and is accompanied by neural differentiation of the cells. $\mathrm{J}$ Pharmacol Sci. 2013;121:25-38.

37 Yamamoto H, Fujimiya M, Shirai Y, Nakashita M, Oyasu M, Saito N. Immunohistochemical localization of serotonin transporter in normal and colchicine treated rat brain. Neurosci Res. 1998;32:305-312.

38 Kang HL, Benzer S, Min KT. Life extension in Drosophila by feeding a drug. Proc Natl Acad Sci U S A. 2002;99:838-843.

39 Wright JM, Zeitlin PL, Cebotaru L, Guggino SE, Guggino WB. Gene expression profile analysis of 4-phenylbutyrate treatment of IB3-1 bronchial epithelial cell line demonstrates a major influence on heat-shock proteins. Physiol Genomics. 2004;16: 204-211.

40 Mori K. Tripartite management of unfolded proteins in the endoplasmic reticulum. Cell. 2000;101:451-454. 